\title{
Output uncertainty of dynamic growth models: Effect of uncertain parameter estimates
} on model reliability

\author{
Anane, Emmanuel; López C, Diana C.; Barz, Tilman; Sin, Gürkan; Gernaey, Krist V.; Neubauer, Peter; \\ Cruz Bournazou, Mariano Nicolas
}

\author{
Published in: \\ Biochemical Engineering Journal
}

Link to article, DOI:

10.1016/j.bej.2019.107247

Publication date:

2019

Document Version

Peer reviewed version

Link back to DTU Orbit

Citation (APA):

Anane, E., López C, D. C., Barz, T., Sin, G., Gernaey, K. V., Neubauer, P., \& Cruz Bournazou, M. N. (2019). Output uncertainty of dynamic growth models: Effect of uncertain parameter estimates on model reliability. Biochemical Engineering Journal, 150, [107247]. https://doi.org/10.1016/j.bej.2019.107247

\section{General rights}

Copyright and moral rights for the publications made accessible in the public portal are retained by the authors and/or other copyright owners and it is a condition of accessing publications that users recognise and abide by the legal requirements associated with these rights.

- Users may download and print one copy of any publication from the public portal for the purpose of private study or research.

- You may not further distribute the material or use it for any profit-making activity or commercial gain

- You may freely distribute the URL identifying the publication in the public portal 


\section{Accepted Manuscript}

Title: Output uncertainty of dynamic growth models: effect of uncertain parameter estimates on model reliability

Authors: Emmanuel Anane, Diana C. López C., Tilman Barz, Gurkan Sin, Krist V. Gernaey, Peter Neubauer, Mariano Nicolas Cruz Bournazou

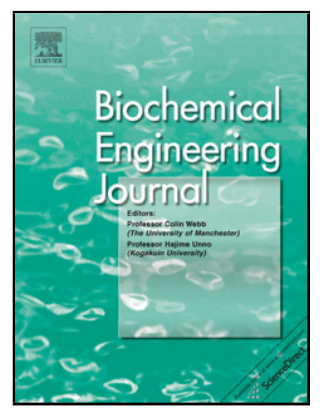

PII: S1369-703X(19)30174-3

DOI: https://doi.org/10.1016/j.bej.2019.107247

Article Number: 107247

Reference:

BEJ 107247

To appear in:

\section{Biochemical Engineering Journal}

Received date: $\quad 8$ February 2019

Revised date: $\quad 27$ March 2019

Accepted date: $\quad 26$ May 2019

Please cite this article as: Anane E, López C. DC, Barz T, Sin G, Gernaey KV, Neubauer P, Cruz Bournazou MN, Output uncertainty of dynamic growth models: effect of uncertain parameter estimates on model reliability, Biochemical Engineering Journal (2019), https://doi.org/10.1016/j.bej.2019.107247

This is a PDF file of an unedited manuscript that has been accepted for publication. As a service to our customers we are providing this early version of the manuscript. The manuscript will undergo copyediting, typesetting, and review of the resulting proof before it is published in its final form. Please note that during the production process errors may be discovered which could affect the content, and all legal disclaimers that apply to the journal pertain. 
Output uncertainty of dynamic growth models: effect of uncertain parameter estimates on model reliability

Emmanuel Anane ${ }^{1}$, Diana C. López C. ${ }^{1}$, Tilman Barz ${ }^{2}$, Gurkan Sin ${ }^{3}$, Krist V. Gernaey ${ }^{3}$, Peter Neubauer ${ }^{1}$, Mariano Nicolas Cruz Bournazou*1

${ }^{1}$ Bioprocess Engineering, Institute of Biotechnology, Technische Universität Berlin, Berlin, Germany

${ }^{2}$ Department of Energy, Austrian Institute of Technology GmbH, Vienna, Austria

${ }^{3}$ Process and Systems Engineering Centre (PROSYS), Department of Chemical and Biochemical Engineering, Technical University of Denmark, Lyngby, Denmark

*Corresponding Author:

Dr.-Ing. Mariano Nicolas Cruz Bournazou, Bioprocess Engineering, Institute of Biotechnology, Technische Universität Berlin, Ackerstraße 76, ACK24, 13355 Berlin, Germany.

Phone: +493031472626

Email: nicolas.cruz@mailbox.tu-berlin.de 


\title{
Highlights
}

- A framework for uncertainty analysis of biokinetic models is presented

- Parameter identifiability is deduced from the dynamic sensitivity matrix

- A mechanistic model of Escherichia coli was analyzed for parameter identifiability and uncertainty of model outputs

- Model prediction accuracy is improved up to 15 times, after regularization.

\begin{abstract}
Mechanistic models are simplifications of bio-physical systems, for which the true values of the model parameters are sometimes unknown. Therefore, before using model-based predictions to study or improve a process, it is essential to ensure that the outputs of the model are reliable.
\end{abstract}

This paper covers the development and application of a framework for practical identifiability and uncertainty analyses of dynamic growth models for bioprocesses. By exploring the numerical properties of the sensitivity matrix, a simple algorithm to determine the presence of non-identifiable parameters in models with high output uncertainty is presented. The framework detects the existence of non-identifiable parameters within the model and proposes a regularisation technique, in conjunction with Monte Carlo Analysis. As an example, the framework was used to analyse a macro-kinetic growth model of Escherichia coli describing a fed-batch process. The results show a reduction in the uncertainty of model outputs from a maximum coefficient of variation of $748 \%$ to $5 \%$ after regularization, and a 15 -fold improvement in the accuracy of model predictions for two independent validation datasets. The presented framework aims to improve the reliability of model predictions and promote a more thorough handling of dynamical models to extend their use in biotechnology. 


\section{Keywords}

Parameter identifiability; Escherichia coli; Fed-batch; Ill-conditioning analysis; Uncertainty analysis; Modelling

\section{Nomenclature}

$\mathrm{K}_{\mathrm{ap}} \quad=$ Monod-type saturation constant, intracellular acetate production

$\mathrm{K}_{\mathrm{sa}} \quad=$ Affinity constant, acetate consumption $(\mathrm{g} / \mathrm{L})$

$\mathrm{K}_{\mathrm{s}} \quad=$ Affinity constant, glucose consumption (gglu./L)

$\mathrm{K}_{\mathrm{ia}}=$ Inhibition constant, inhibition of cellular growth by extracellular acetate

$\mathrm{K}_{\text {is }} \quad=$ Inhibition constant, inhibition of acetate uptake by glucose $(\mathrm{g} / \mathrm{L})$

$\mathrm{p}_{\mathrm{Amax}}=$ Maximum specific acetate production rate $\left(\mathrm{g} \_\right.$ace $\left.\left./(\mathrm{gx} . \mathrm{h})\right)\right)$

$\mathrm{q}_{\mathrm{Amax}}=$ Maximum specific acetate consumption rate (g_ace/(gx.h)))

$\mathrm{q}_{\mathrm{m}} \quad=$ Specific maintenance coefficient $(\mathrm{g}$ _glu/(gx.h))

$\mathrm{q}_{\text {Smax }}=$ Maximum specific glucose uptake rate (glu./gx.h)

$\mathrm{Y}_{\mathrm{as}}=$ Yield of acetate on substrate (g.ace/g.glu)

$\mathrm{Y}_{\mathrm{oa}}=$ Specific oxygen used per gram of acetate metabolised $\mathrm{g} / \mathrm{g}$ )

$\mathrm{Y}_{\mathrm{xa}} \quad=$ Yield of biomass on acetate

$\mathrm{Y}_{\mathrm{em}}=$ Yield of biomass on glucose, excluding maintenance $\left(\mathrm{Y}_{\mathrm{em}}=\mathrm{Y}_{\mathrm{xs}}-\mathrm{q}_{\mathrm{m}}\right)$

$\mathrm{Y}_{\mathrm{os}}=$ Oxygen used per gram of glucose metabolised per gram biomass $(\mathrm{g} / \mathrm{g})$

$Y_{\mathrm{xsof}}=$ Yield of biomass on all other products of overflow routes, excluding acetate

$\mathrm{C}=$ Carbon content of $(\mathrm{s})$ substrate, $(\mathrm{x})$ biomass

DOT $=$ dissolved oxygen tension

$\mathrm{F} \quad=$ Flow rate $(\mathrm{L} / \mathrm{h})$

$\mu \quad=$ specific growth rate $\left(\mathrm{h}^{-1}\right)$ 


\subsection{Introduction}

The contribution of mathematical models to our understanding of biological systems from Monod [1] and Andrews [2] to bioprocess engineering [3], biochemical systems [4], systems biology [5], metabolic engineering [6], flux balance analysis [7], synthetic biology [8] and bioinformatics [9] is undisputed. The reader is referred to Jay Bailey [10] for a critical overview of the development, application and potential of mathematical models in biotechnology. Unfortunately, the usability of models in biological systems seems to be underestimated, showing only a slow advance in tackling the challenges that were stated two decades ago [10]. There is a general consensus that all the model based tools for advanced engineering are currently not applicable in the bio-industry [11].

One of the major obstacles hampering the use and acceptance of mechanistic models in bioprocesses is the lack of simple reliability tests for the predictions of a model. Especially in bioengineering, first principles models need to describe large, highly nonlinear and state dependent processes using simple equations and lumped kinetic parameters. Due to the complexity of the underlying metabolic and physiological phenomena, a large number of parameters is typically required to characterise a given biological system. Routinely, a validation of the mechanistic model is claimed by presenting the outputs of the model (at some estimated parameter values) showing a good agreement with experimental data, usually within a certain experimental range. But, by this, the reliability of the parameter estimates is not considered; a typical issue being the existence of infinite combinations of parameter values that can show the same fit (a non-identifiable parameter set). Whereas data-based models are categorically rejected if not presented with its associated confidence regions (e.g. error bars), mechanistic models are rarely shown with similar reliability tests [12]. More importantly, one of the major advantages of mechanistic models is their ability to predict the systems behaviour beyond the region of experimentation (e.g. in scale-up), so that it is crucial to evaluate its predictive power with sound reliability assessment methods. Today, many mechanistic biological models are published without testing their structural and practical identifiability, the uncertainty of parameter estimates after fitting, and the propagation of this uncertainty on model outputs. The reason for this is not a lack of sufficient literature focused on parameter identifiability of mechanistic 
models. There are numerous papers and reviews on the topic for both biosystems [13] and general mechanistic models [14-16]. Additionally, software packages, such as Amigo2 [17] and BioPreDyn suite [18] have been developed as generic plug-and-play platforms for advanced analysis, including identifiability and sensitivity analyses especially suited for systems biology models. Whereas these works and packages are suitable for application by advanced modellers, the complex mathematical concepts presented in them are usually beyond the academic training and mathematical background of biotechnologists and bioprocess engineers $[19,20]$. Furthermore, though there are procedures to select appropriate modelling methodologies, there are many methods at different levels of complexity with their respective advantages and disadvantages, so that it is very easy to get lost in the search of a simple, reliable and thorough methodology to test the reliability of parameter estimates.

The aim of this paper is to present a comprehensive framework for the analysis of the reliability of the outputs of a given nonlinear dynamical model, specifically directed at non-experts in the field. We deal with this issue from the point of view of an engineer, biotechnologist or a molecular biologist with limited training in these mathematically loaded methods. As an example, we apply this strategy to analyse a macro-kinetic growth model of Escherichia coli, and the effect of an ill-conditioned parameter estimation on model predictions assessed by output uncertainty quantification. In another dimension, and to the best of our knowledge, this is the first time a mechanistic model of $E$. coli has been analysed for the identifiability of its parameters and the reliability of its predictions, although these models have been used in biotechnology for several decades. With this framework, we aim to promote a more thorough handling of relatively complex first principles models in biotechnology so as to increase their reliability and acceptance.

\subsection{Reliability of model outputs}

The outputs $y$ of a dynamical model (Equation 1) depend on the initial values of the state variables $x_{0}$, the experimental conditions $u$, and the parameter vector $\theta$. Assuming the structure of the model is correct with random experimental errors and knowing $x_{0}$ and $u$ (already a strong statement), we can confine the parameter values to some region of confidence. Thus, since we do not know the exact values of $\theta$, and $\theta$ 
influences $y$, we know that the outputs of our simulation $y$ must entail some uncertainty.

For the sake of clarity, we consider that we can describe the process of interest by a mathematical model consisting of a non-linear Ordinary Differential Equation (ODE) system with the following characteristics:

$y(t)=A(x(t))$

$\dot{x}(t)=f(x(t), u(t) ; \theta)$

$x\left(t_{0}\right)=x_{0}$,

where $t$ is the independent variable time, $x(t) \in \mathbb{R}^{n_{x}}$ is the vector containing the dependent state variables. $\theta \in \mathbb{R}^{n_{p}}$ is the unknown parameter vector (with $n_{p}$ parameters), $u(t) \subseteq U \in \mathbb{R}^{\mathrm{n}_{\mathrm{u}}}$ contains the time-varying input signals (i.e. experimental design variables). The initial conditions are given by $x_{0} \in X_{0}$, where $y(t) \in \mathbb{R}^{n_{y}}$ is a vector of predicted output (response) variables. The measured state variables, $y(t)$, comprising both on-line ( $\mathrm{y}_{\text {on }}$ ) and off-line ( $\mathrm{y}_{\mathrm{of}}$ ) measurements, is a subset of $\mathrm{x}(\mathrm{t})$, defined by the constant selection matrix $A \in \mathbb{R}^{n_{y} \cdot n_{x}}$.

The uncertainty on $y(t)$ depends on the uncertainty of $\theta$ and on how changes in $\theta$ are propagated throughout the model. For a given setup, $x_{0}$ and $u(t)$, we can compute a point estimate of the model parameters in $\theta$ by fitting it to experimental data (performing a Parameter Estimation PE). The important question is: how precisely can we estimate $\theta$ at the $x_{0}$ and $u(t)$ defined in our experiments, so that $y(t)$ has low uncertainty even at different conditions of $x_{0}$ and $u(t)$ defined by e.g. a different reactor or the industrial process at different scale?

We can calculate some approximations to answer this question and for this we need to find out two things i) the identifiability of $\theta$ (how large is the confidence interval of the estimates of $\theta$ after the PE) and ii) how does this uncertainty on $\theta$ affect the reliability of the outputs $y$ at different conditions. 


\subsection{Some existing methods for parameter identifiability analysis}

Parameter identifiability analysis is used to test whether a unique estimate within a confidence region can be assigned to the parameter set of a model after parameter estimation from experimental data. The model is said to be structurally non-identifiable [20-22] when it has inherently redundant parameters that cannot be uniquely estimated independently from the observations available. On the other hand, if some parameters are non-identifiable due to limitations in the quantity or quality of the data (information content of the data), the model is considered to be practically non-identifiable [23]. Whereas structural non-identifiability can be addressed through model reduction [24], practical non-identifiability can be addressed by improving the data quality (performing more informative experiments [25] or (at the cost of a higher bias) by regularization of the parameter estimation problem (e.g. Tikhonov [26] or singular value truncation [27]).

There are several methods to analyse the identifiability of a model. The methods for structural identifiability analysis include, among others, the Taylor series expansion, Lie derivatives and differential algebra. The reader is referred to the review by Chis and colleges [28] for a comprehensive treatment of some of these methods.

Practical identifiability methods include likelihood profiling, eigenvalue decomposition of the Fisher Information Matrix (FIM) [29] and various forms of combinatorial methods that rank parameter subsets according to practical identifiability using the properties of the eigenvalues [15,30,31].

In this work, we focus on the case where i) the model is at hand and has been developed using a priori knowledge of the system (e.g. cell physiology, metabolomics) and ii) the reliability of the existing model at its "true" parameter values is of interest. Hence, the aim is to provide a comprehensive framework for practical (local) identifiability of biochemical models by studying the numerical properties of the model sensitivity matrix.

\subsection{Model analysis framework}

The framework comprises three major tasks (Figure 1): (i) ill-conditioning diagnosis, (ii) regularization to handle ill-conditioned problems and (iii) propagation of the 
parameter uncertainty onto model outputs (output uncertainty analysis). The model analysis technique presented in Figure 1 is similar to the method of eigenvalue decomposition of FIM by Quaiser and Mönnigmann (2009). However, in order to circumvent problems related to the calculation of an ill-conditioned FIM, it uses the singular value decomposition (SVD) of the sensitivity matrix [32] and further includes a Monte Carlo based method to quantify parameter uncertainty if the problem is illconditioned. This is a local analysis of the sensitivity matrix (the results are dependent on the parameter values). All these steps are preceded by data collection from experimental runs and a preliminary parameter estimation. The details of the workflow in the model analysis framework are discussed in the following sections.

\subsection{Parameter Estimation (PE)}

During parameter estimation, the true parameter values of the model are inferred by minimizing the difference between the observed data and the model predictions. The parameters $\theta$ of the dynamical model are typically estimated by solving the optimization problem of the form:

$\hat{\theta}:=\arg \min _{\theta} \Phi(U, \theta)$

where the objective function $\Phi(U, \theta)$, which in this case is the weighted nonlinear leastsquares function between the model predictions $Y(U, \theta)$ and the experimental data $Y^{m}$, is calculated using the maximum likelihood estimator:

$\Phi(U, \theta):=\frac{1}{2}\left(Y(U, \theta)-Y^{m}\right)^{T} \mathrm{C}_{\mathrm{y}}^{-1}\left(Y(U, \theta)-Y^{m}\right)$

In Equation 3, all the measured data collected at the experimental conditions $\left(x_{0}, u\right)$ are compiled in the vector $Y^{m}$. To take the measurement errors into account during parameter estimation, the objective function is weighted by the variance-covariance matrix, $C_{y}$ which in our case is obtained assuming unbiased, independent and normally identically distributed measurement errors. Therefore $C_{y}$ is a diagonal matrix with entries given by the variance $\sigma_{y}^{2}$ of each measurement.

\subsection{Sensitivity Matrix}


The identifiability of a parameter set (how accurate can we estimate the values of the parameters) is defined by two properties: (i) parameter sensitivity: the effect this parameter has on the objective function; and, (ii) parameter correlation: how correlated this effect is with the effects of the other parameters. Hence, we obtain useful information about how well we can estimate the parameters by analysing the properties of the sensitivity matrix $S$ Equation 4 [33] computed from the first order partial derivatives of the state variables (y) with respect to the parameters,

$S=\left[\begin{array}{cccc}\frac{\partial y_{1}}{\partial \theta_{1}} & \frac{\partial y_{1}}{\partial \theta_{2}} & \cdots & \frac{\partial y_{1}}{\partial \theta_{N_{\theta}}} \\ \vdots & \cdots & \vdots \\ \frac{\partial y_{N v}}{\partial \theta_{1}} & \frac{\partial y_{N v}}{\partial \theta_{2}} & \cdots & \frac{\partial y_{N v}}{\partial \theta_{N_{\theta}}}\end{array}\right]$

where $N_{v}$ is the number of state variables and $N_{\theta}$ is the number of parameters. Since the analysis considers dynamical models, $\mathrm{S}$ is time-variant. The sensitivity matrix can be calculated in a simple way with the method of finite differences (i.e. perturbation method) [34]. Nevertheless, the computational burden increases exponentially with the number of parameters and has inherent inaccuracies associated with the numerical integrator and the magnitude of parameter perturbations $(\Delta \theta)[35]$.

In the forward method, the dynamics of the sensitivity functions along the time vector are given by $[23,36]$

$\frac{d S}{d t}=\frac{\partial f}{\partial y} S+\frac{\partial f}{\partial \theta}$

where $\frac{\partial f}{\partial y}$ represents the Jacobian of the system w.r.t the state variables and $\frac{\partial f}{\partial \theta}$ is the Jacobian w.r.t the parameters. The dynamic sensitivity matrix $S$ of the measured state variables $Y$ is obtained by integrating Equation 5 together with the model equations, with the initial condition $\mathrm{S}\left(\mathrm{t}_{0}\right)=\frac{\partial f\left(t_{0}\right)}{\partial \theta}$.

$\mathrm{S}=\left[\left.\left.\left.\frac{\partial Y}{\partial \theta_{1}}\right|_{t 1} \frac{\partial Y}{\partial \theta_{1}}\right|_{t 2} \cdots \frac{\partial Y}{\partial \theta_{1}}\right|_{t n}\right]^{T}$

The Symbolic Math Toolbox in Matlab, Maple, or Sympy among others can be used to calculate the partial differentials of Y w.r.t $\theta$, and these can be integrated together with the model equations to obtain the dynamic sensitivity matrix S. The non-dimensional 
sensitivity matrix $\tilde{S}$ is obtained by normalizing $\mathrm{S}$ with the nominal parameter and model output values [37]. Since the sensitivity matrix is computed at the given parameter values and not at all the possible parameter combinations, the analysis in this framework is local in nature.

\subsection{Ill-conditioning Diagnosis}

The PE is an optimization problem with no warranty to have a unique solution (a necessary condition for well-posedness). That is, if there exist various parameter combinations that deliver similar values of the objective function $\Phi$, the problem is said to be ill-posed, hence causing ill-conditioned numeric approximation. Practically, this means that very large values are divided by very small ones during the numerical computation of the parameter estimates, causing large inaccuracies and instability in the results. When the PE problem is ill-conditioned, it leads to overestimation of the variance of the parameter estimates. Additionally, the experimental data are inherently subject to errors. Hence, it is important to determine how the conditioning of the parameter estimation problem and measurement errors translate into parameter uncertainty, even for well-posed problems. The analysis presented in the current framework to carry out ill-conditioning diagnosis is based on the orthogonal decomposition of the sensitivity matrix $\tilde{S}$. This decomposition, namely singular value decomposition (SVD) is used to calculate the rank of $\tilde{S}$, used to detect linear dependences that result in a non-invertible Fisher-information and Jacobian matrices (making the estimation ill-conditioned). The $N_{Y}-b y-N_{\theta}$ sensitivity matrix $\tilde{S}$ is decomposed according to Equation 7

$\tilde{S}=U \Sigma V^{\prime}$

where $\mathrm{U}\left(N_{Y^{-}}-b y-N_{Y}\right)$ and $\mathrm{V}\left(N_{\theta}-b y-N_{\theta}\right)$ are orthogonal unitary matrices and $\Sigma$ is an $N_{Y^{-}}$ by- $N_{\theta}$ diagonal matrix with ordered non-negative diagonal elements $\varsigma_{11} \geq \varsigma_{22} \geq$ $\varsigma_{33} \ldots \geq \varsigma_{N_{\theta}}$, which are the singular values of $\tilde{S}[38,39]$. These singular values are then used to calculate two metrics [40]: the condition number $(\kappa)$ and collinearity index $(\gamma)$, as

$\kappa_{i}=\frac{\varsigma_{\max }}{\varsigma_{i}} \quad$ and $\quad \gamma_{i}=\frac{1}{\varsigma_{i}}$ 
The condition number $(\kappa)$ of parameter $i$ is a measure of the sensitivity of the model outputs to perturbations in this parameter, whereas the collinearity index is a measure of the linear independence of the parameter [15]. The numerical rank $r_{\epsilon}$ is the number of singular values that are greater than $\epsilon$, where $\epsilon$ is a threshold defined by the combined empirical conditions

$$
\epsilon:=\kappa_{i} \leq \kappa_{\max } \Lambda \gamma_{i} \leq \gamma_{\max }
$$

The limits $\kappa_{\max }$ and $\gamma_{\max }$ are empirical thresholds determined as 1000 and 15, respectively, according to the works of Grah [40] and Brun [15]. The matrix is said to be rank-deficient, indicating a covariance matrix close to singular and an ill posed PE, if $r_{\epsilon}<N_{\theta}$, where $N_{\theta}$ is the number of parameters.

\subsection{Regularization}

If the PE turns out to be ill-posed $\left(r_{\epsilon}<N_{\theta}\right)$, there are several options and methods to regularize the problem (at the cost of adding a priori information). If the issue is structural identifiability, methods that range from sophisticated mathematical tools to broad simplifications in model structure exist to reduce the number of states and parameters $[22,41]$. On the other hand, to deal with practical identifiability the first step is to improve the experiments so as to increase the information content of the data [25]. Still, when technical limitations hamper informative experiments, the PE problem can be regularized to reduce the inflation of the uncertainty in the parameters by adding $a$ priori knowledge. This a priori knowledge can be in the form of expert knowledge, experimental data from other sources or literature data. With the emergence of online repositories for quantitative biological data, such as BioNumbers [42], BRENDA [43] and Elsevier BioBase, it is becoming increasingly easier to find quality biological data on reaction kinetics, yield coefficients, metabolite concentrations, etc. with sound references, which can be used as a priori information for regularization.

(i) Tikhonov Regularization and Monte Carlo Analysis: This is a more formal way of getting around ill-conditioned parameter estimation problems. In the most common form of this method, the ill-conditioned parameter estimation problem is made regular by re-defining the objective function (Equation 3) with the addition of a penalty term, $\Gamma(\theta)$ in quadratic form [26]. 
$\Gamma(\theta)=\left(\theta^{*}-\theta\right)^{2}$

(10).

In equation $10, \theta^{*}$ represents the prior information (literature values of parameters) whereas $\theta$ is the vector of current parameter values. The penalization term is weighted by a sufficiently large $\lambda$ so that the objective function $\Phi(U, \theta)$ can always be inverted.

$\Phi(U, \theta):=\frac{1}{2}\left(Y(U, \theta)-Y^{m}\right)^{T}\left(C_{y}\right)^{-1}\left(Y(U, \theta)-Y^{m}\right)+\lambda \Gamma(\theta)$

This penalty term, which is a non-singular symmetric matrix and has a minimum at the initial parameter values, contains prior information (e.g. literature values) of the parameters [16]. The contribution of the prior information is inevitably associated with bias so that the influence of the regularization should be kept as small as possible. The weighting factor $(\lambda)$ in the penalty term determines whether more emphasis is placed on the data or on the prior knowledge of the system during PE. However, the choice of a weighting factor (tuning the regularization) to have proper balance between the data and the prior information is not a trivial task, and is beyond the scope of the current contribution. The interested reader is referred to the work of Gábor and Banga (2015) [36] for a comprehensive treatment of different iterative methods for tuning the weighting factor.

In the current contribution, the Tikhonov technique is combined with a Monte Carlo (MC) procedure to efficiently regularize ill-conditioned parameter estimation problems. The MC method is a more mathematically efficient way to accurately quantify the non-linear correlations inherent in a model, which are useful in estimating the output uncertainty of the model. In the method, a total of $n$ in-silico datasets are generated by random sampling with replacement (Bootstrap) from the measurement errors [37]. The bootstrap sampling technique works well without requiring the assumption of normal distribution of the measurement errors [44]. Each set of the insilico data is used to carry out regularized $\mathrm{PE}$, resulting in a matrix of parameter estimates $\theta_{m c}(n-b y-N \theta)$, from which the mean values of the parameters are calculated. At the mean values $\left(\bar{\theta}_{m c}\right)$, both the sensitivity matrix and its rank $\left(r_{\epsilon}\right)$ are re-calculated. If the problem is still ill-conditioned $\left(r_{\epsilon}<N_{\theta}\right)$, the MC parameter estimation is repeated with a fine-tuned regularization (adjusting $\lambda$ ); otherwise the model calibration 
is completed and the raw MC results $\left(\theta_{m c}\right)$ can be used for further output uncertainty analysis.

(ii) Subset Selection ( $\mathrm{SsS}$ ) with Empirical Knowledge: As opposed to black box models, mechanistic ones entail empirical knowledge, although this knowledge is sometimes difficult to completely define in mathematical equations. Furthermore, the experimental conditions or the observations may be restricted due to practical constraints impeding the estimation of certain parameters that are known to be relevant. Therefore, regularizing the problem by assigning fixed values to non-identifiable parameters based on a priori information is a very efficient method (when properly performed), although the bias caused by a priori information and subjective decisions should be kept minimal. In application, the non-identifiable parameters are taken out of the PE problem using subset selection techniques and given fixed values. It should be noted that the subset selection process considers both linear independence of the parameters and the sensitivity of model outputs to each parameter. The parameters in the identifiable set simultaneously satisfy both conditions for sensitivity and linear independence, set by the thresholds $\kappa_{\max }$ and $\gamma_{\max }$. Therefore, it is possible that model output may be highly sensitive to a parameter in the non-identifiable, but this parameter was rejected in the SsS process due to correlation to one or more of the parameters in the identifiable set. Great care must be taken when fixing numerical values for such sensitive yet non-identifiable parameters [15] during subsequent parameter estimation as these parameters have a strong influence on the estimated parameter values. A sound knowledge of the process or the biological system being modelled is very important in setting the non-identifiable parameters to fixed values that make sense both physiologically and in process design. This manual regularization technique can be a powerful tool, for example in macro-kinetic models of biological systems, where there is a huge wealth of knowledge. An example of implementation of this technique is given in the Supplementary material.

\subsection{Propagation of Parameter Uncertainty onto Model Outputs}

When computing the outputs of the model with the parameter estimates which have some level of uncertainty, we need to consider all possible outputs within this parameter 
space. That is, we must consider that the true parameters lie somewhere inside the confidence region of the estimates. The projection of the uncertainties associated with the parameter estimation $\left(\bar{\theta}_{m c}\right.$ or $\left.\hat{\theta}\right)$ to the model output space and the associated quantification of the output variance should be the determining factor to assess the usability of the model. This output uncertainty can be analysed through a Monte Carlo procedure (MC-2) where various parameter combinations from a defined space are used to run model simulations.

The first step is to define the parameter space. For models with well-posed PE problems $\left(r_{\epsilon}=N_{\theta}\right)$, the parameter input space is defined by the confidence intervals of the parameters. For such problems, the variances of the parameter estimates, $\sigma_{\theta_{i}}^{2}$ (and hence the standard deviations, $\sigma_{\theta_{i}}$ ) are given by the diagonal elements of the covariance matrix $\operatorname{Cov}(\hat{\theta})$, which is calculated by a first order linear approximation method,

$\operatorname{Cov}(\theta) \approx s^{2}\left[\tilde{S} \cdot \tilde{S}^{T}\right]^{-1}$

where $s^{2}$ is the unbiased variance of parameter estimates [37]. The correlation coefficient between any two parameters $\left(\theta_{l}\right.$ and $\left.\theta_{k}\right)$ can then be calculated from the covariance matrix as

$\operatorname{Corr}\left(\theta_{l}, \theta_{k}\right)=\frac{\operatorname{Cov}\left(\theta_{l}, \theta_{k}\right)}{\sqrt{\sigma_{\theta_{l}}^{2}} \sigma_{\theta_{k}}^{2}}$

The confidence intervals $[l b u b]$ of the estimated parameters are calculated using Student $t$-distribution at 95\% confidence level [37]. In the second step, $n$ independent random samples are generated from $\left[\begin{array}{ll}l b & u b\end{array}\right]$ using the Latin hypercube sampling algorithm. The correlation matrix is then used to transform these samples into quasirandom, dependent samples; to depict the inherent interdependencies among model parameters resulting from fundamental theories (e.g. Monod kinetics, inhibition, Michaelis-Menten) [45,46]. In the third step, each set of quasi-random parameter samples is used to carry out model simulations. The mean and standard deviation of the resulting model outputs are then calculated as a measure of the level of uncertainty associated with model predictions. 
For ill-conditioned problems, the parameter space is already defined in the MC parameter estimations. The matrix of parameter estimates $\theta_{m c}(n-b y-N \theta)$ contains the real, non-linear correlations of the model parameters as well as the real distribution of the values each parameter can take, with the given data. Thus, for uncertainty analysis, each set of parameter estimates from $\theta_{m c}$ is used to run model simulations, from which the mean and standard deviations of the outputs are calculated to quantify the uncertainty of model predictions.

\subsection{Case Study：Macro-kinetic Growth Model of $E$. coli}

To illustrate the use of the proposed framework, we analyse a mechanistic model of $E$. coli cultivated in a fed-batch process. Although such models have been widely used for both bioprocess development and for elucidation of various physiological concepts in E. coli, this is the first time a mechanistic model describing E. coli physiology is analysed with such methods to ascertain the reliability of the model predictions.

\subsection{E. coli Model Description}

The model describes general growth of $E$. coli and the use of glucose based on the substrate partitioning concept $[47,48]$. It describes metabolic pathways of glucose uptake and its subsequent conversion to cellular material in anabolic routes, its use for energy generation or its conversion to acetate through overflow metabolism. The interested reader is referred to the referenced works [49-52] for a detailed description of the model, including its physiological basis. The model comprises 4 state variables describing biomass, glucose, acetate and dissolved oxygen profiles (i.e. $\mathrm{x}=[\mathrm{X}, \mathrm{S}, \mathrm{A}$ and DOT]) in $E$. coli cultivations, with a total of 15 unknown parameters $\left(N_{\theta}=15\right.$, Table 1).

\subsection{Fermentation Data}

The data used for this case study was obtained from a fed-batch cultivation of $E$. coli W3110 in a 3.7 L BioEngineering ${ }^{\circledR}$ bench top bioreactor. The cultivation temperature was $37{ }^{\circ} \mathrm{C}$ whilst the $\mathrm{pH}$ was controlled at a set point of 7.0. For the batch phase, $2 \mathrm{~L}$ of medium was inoculated with an appropriate volume of the pre-culture to $\mathrm{OD}_{600}$ of 0.1 and initial glucose concentration of $5 \mathrm{~g} / \mathrm{L}$. The exponential fed-batch phase started with $300 \mathrm{~g} / \mathrm{L}$ glucose solution to maintain a set specific growth rate $\left(\mu_{\text {set }}\right)$ of $0.22 \mathrm{~h}^{-1}$. 
After 3 hours, the feed was switched to a constant feed, where the last value of the exponential feed profile was maintained for a period of 10 hours. Intermittent glucose pulses were given during the constant feeding phase to explore the dynamics of glucose uptake and acetate re-assimilation in E. coli. The measured state variables during the cultivation were dissolved oxygen (with a polarographic DO probe), biomass, acetate and residual glucose. The interested reader is referred to Anane et al [51] for further details on the experimental procedures. For model validation, two other independent cultivations were carried out in the same bioreactor using the same protocol, but with significant differences in the cultivation scheme from the parameter estimation dataset. The cultivation for the first validation data was a simple batch followed by exponential fed-batch, without a constant feeding phase. The second cultivation for model validation comprised a batch phase, followed by two exponential fed-batch phases with different slopes $\left(\mu_{\text {set }}=0.32 \mathrm{~h}^{-1}\right.$ and $0.15 \mathrm{~h}^{-1}$ ), which are distinct growth phases of overflow and non-overflow conditions, respectively, for E. coli. Additionally, two intermittent glucose pulses were given in the second exponential feeding phase to trigger overflow conditions within the slow growth phase. Since the model was calibrated and analysed at a different $\mu_{\text {set }}$ value, it was of interest to see how it will perform in predicting conditions of distinct overflow phases (e.g. low $\mu_{\text {set }}$ ), which are prevalent and important in E. coli process design and operation.

\subsection{Parameter Estimation using Fed-batch Data}

The computation results of the state variables that describe growth profiles of $E$. coli in the course of the cultivation together with their corresponding measured values are shown in Figure 2. The PE problem (Equation 2) was solved using the lsqnonlin function in $\operatorname{Matlab}^{\circledR}$, and the optimal solution for $(\hat{\theta})$ is given in Table 1 . It is noteworthy that, at the optimal parameter values, the model is able to track rapid changes in experimental conditions, such as the intermittent glucose pulses in the constant feed fed-batch phase (Figure 2). Without further analysis, the model seems to properly fit the data at the estimated parameter values. But, as will be seen in the identifiability analysis, this result is inside a large region of possible outputs, and is therefore not unique.

\subsection{Ill-conditioning Diagnosis}


A singular value decomposition (Equation 7) was computed at the parameter estimates $(\hat{\theta})$, using the normalized sensitivity matrix of the model outputs with respect to the parameters. The thresholds of 15 and 1000 for the maximum collinearity index $\left(\gamma_{\max }\right)$ and condition number $\left(\kappa_{\max }\right)$, respectively, were selected as defined in Equation 9. A plot of the singular value spectrum, together with the thresholds is shown in Figure 3. Using the thresholds, a numerical rank $r_{\epsilon}=10$ was determined for the sensitivity matrix at $\hat{\theta}$, which means only 10 out of the 15 columns of $\tilde{S}$ were linearly independent and sensitive enough to satisfy the conditions of the thresholds.

\subsection{Regularization and Monte Carlo Analysis}

Since the PE problem is ill-posed on $\hat{\theta}$ according to the $\operatorname{SVD}\left(r_{\epsilon}<N_{\theta}\right)$, we must regularize the problem by introducing some a priori information on the parameters. Thus, literature values of the parameters (initial guess) were incorporated into the objective function as given in Equation 10, to regularize subsequent parameter estimation runs. A regularization parameter $(\lambda)$ of 311.6 was determined as the optimal value from a range of $10^{6}$ to $10^{-4}$, using the L-curve method (see Appendix for details of tuning the regularization). A total of 500 in-silico, Monte Carlo (MC) datasets, $Y^{m c}$ were then randomly generated from the measurement errors (standard deviations of triplicate measurements for biomass, acetate and glucose) using bootstrap sampling with replacement. For high precision measurements such as DOT, the standard deviation was assumed to be $5 \%$ of the measurements. The MC datasets were then used to run 500 regularized parameter estimations to yield the matrix $\theta_{m c}$. The mean and standard deviations of the MC estimates are given in Table 1, whereas a matrix plot of $\theta_{m c}$ is shown in Figure 4, with the distribution of estimates for each parameter on the diagonal subplots. An extended view of the probability distribution of the parameter estimates is shown in Figure 4B, where the actual distribution of the estimates compared with the assumed normal distribution are also shown. The more narrow peaks ( $\mathrm{q}_{\mathrm{Smax}}, \mathrm{q}_{\mathrm{m}}, \mathrm{Y}_{\mathrm{xsof}}$ ) indicate parameters for which most of the estimates converged to a single value, implying that these are highly identifiable, whereas parameters with broad peaks show general non-identifiability with the given data.

The MC datasets represent different experimental scenarios within the region of the current data and the results show both the inherent non-linear and linear correlations 
among the parameters. A careful observation of the samples reveals some important physiological perspectives in the parameter correlation patterns. For instance, the maximum specific acetate re-assimilation rate $\left(q_{\text {Amax }}\right)$ is non-linearly correlated with the acetate inhibition constant $K_{i s}$ (circled with red dotted lines in Figure 4) whilst the maximum substrate uptake rate $\left(q_{\operatorname{Smax}}\right)$ and the biomass yield coefficient $\left(Y_{e m}\right)$ are negatively correlated in a linear manner. This is because at higher $q_{S \operatorname{Smax}}$ values, a greater part of the carbon source is directed to overflow metabolism with by-products that eventually inhibit the cell growth [53], thereby reducing the biomass yield.

\subsection{Output Uncertainty Analysis}

Each of the parameter estimates in $\theta_{m c}$ was used to carry out model simulation, as outlined in Figure 1. The purpose of the regularization is to reduce the variance of the parameter estimates, to improve the accuracy of model predictions for further applications. The major model outputs (biomass, glucose, acetate and dissolved oxygen tension) from the output uncertainty analysis for regularized parameter estimations are plotted in Figure 5B. For comparison, a MC parameter estimations was also carried out (using $Y^{m c}$ ) without regularization, and the results were used for output uncertainty analysis (Figure 5A). For the case without regularization, the maximum coefficient of

variation $\left({ }^{\sigma_{Y}} /_{\bar{Y}} \times 100 \%\right)$ for the predicted model outputs were: $74.4 \%$ for biomass (X), occurring at $6.6 \mathrm{~h}$ within the batch phase, $143.5 \%$ for glucose $(\mathrm{S})$ occurring at the point of the first glucose pulse and $85.1 \%$ for DOT occurring immediately after the first glucose pulse. The prediction error for acetate increased monotonically along the cultivation profile, reaching a maximum of $748.5 \%$ at the end of the cultivation. With the regularized PE by the Tikhonov method, the maximum coefficient of variation for the model outputs was: $3.20 \%$ for biomass (X), $1.06 \%$ for glucose (S), $4.75 \%$ for acetate and $4.62 \%$ for DOT, which is a significant improvement in the prediction quality compared to the base case without regularization. For comparison, the results of the output uncertainty using regularization by subset selection are given in Table A.1 and plotted in Figure A.1 (Supplementary Material). Both regularization methods result in significant improvements in the predictability of model outputs, with low uncertainty, thereby improving the reliability of the model.

\subsection{Model Validation}


Using the mean parameter values of the regularized parameter estimation $\left(\bar{\theta}_{m c}\right)$, the model was used to predict the outcome of the two validation experiments, using the experimental conditions of each dataset. The predictions were then compared to the actual experimental data, as shown in Figure 6. The profiles of the relatively simple experiment of the first validation run were accurately predicted by the model (Figure $6 \mathrm{~A}$ ) using $\bar{\theta}_{m c}$ (except for $\mathrm{K}_{\mathrm{L}}$ a values which were calculated externally from the operating conditions of the bioreactor). As stated earlier, the second validation cultivation was more complex, but the experimental profiles were predicted to acceptable accuracy by the model. In both validation datasets, the least accurately predicted output was the acetate values, where the model seemed to show higher acetate re-assimilation rates than the actual experimental data towards the end of each cultivation. However, the overall trends in acetate profile were accurately predicted (Figure 6B), showing acetate accumulation at higher specific growth rates $\left(\mu_{\text {set }}=0.32\right.$ $\mathrm{h}^{-1}$ ) and its re-assimilation at lower specific growth rates $\left(\mu_{\text {set }}=0.15 \mathrm{~h}^{-1}\right)$. For a quantitative comparison, the relative root mean square error (RRMMSE) of prediction of the validation data was calculated using Equation 14:

$R R M S E=\sqrt{\frac{1}{n} \sum_{i=1}^{n}\left(\frac{Y_{i}(U, \theta)-Y_{i}^{V}}{Y_{i}^{V}}\right)^{2}}$

where $\mathrm{Y}^{\mathrm{V}}, \mathrm{Y}(U, \theta)$ and $n$ represent the validation data, model predictions and number of predicted data points, respectively. Using the traditional parameter estimates $(\hat{\theta})$ and regularized parameter estimates $\left(\bar{\theta}_{m c}\right)$, the RRMSE values improved from $25 \%$ to 1.6 $\%$ for the first data set, whereas a reduction from $41 \%$ to $6.5 \%$ was achieved for the second data set. Thus, the identifiability analysis and regularization resulted in up to 15 times higher accuracy for model predictions than the non-regularized problem.

\subsection{Discussion}

The framework presented in this contribution deals with the identifiability analysis of non-linear growth models in a relatively direct way by calculating the local sensitivity matrix and deducing non-identifiability from the numerical properties of this matrix. This is followed by a knowledge based regularization to handle the ill-posed problem, 
which significantly improves the reliability of model predictions as shown by the 15fold reduction in RRMSE in the validation results of the case study. From the physiological point of view, some parameters that were considered non-identifiable by the framework, such as the maintenance coefficient $q_{m}$, and the apparent inhibition of acetate uptake by glucose $K_{i s}$ are known to be an integral part of the growth parameters of E. coli [58]. Since these parameters are well-documented in literature [42], it is usually easier to obtain physiologically meaningful a priori values for regularization, as discussed by [37]. However, parameters such as the Monod-type saturation constants for acetate production, acetate yield on glucose and biomass production from reassimilation of mixed acid fermentation products are quite obscure and more difficult to describe mechanistically [54], thereby making them non-identifiable with the given data. It is worth noting, also, that the identifiable parameters (see Appendix) include parameters that describe general growth $\left[\mathrm{q}_{\mathrm{Smax}}, \mathrm{Y}_{\mathrm{em}}, \mathrm{Y}_{\mathrm{os}}, \mathrm{K}_{\mathrm{s}}\right.$ ], overflow metabolism [p $\mathrm{p}_{\mathrm{Amax}}, \mathrm{K}_{\mathrm{ia}}, \mathrm{Y}_{\mathrm{oa}}$ ], and acetate re-assimilation in E. coli $\left[\mathrm{q}_{\mathrm{Amax}}, \mathrm{Y}_{\mathrm{xa}}, \mathrm{K}_{\mathrm{sa}}\right.$ ], which are important concepts in the design of $E$. coli fermentation processes[21,55] .

The foregoing analysis is used to improve the prediction quality for experimental scenarios that may lie outside the regime of both the calibration and validation data (such as scale-up calculations from pilot scale data), based on physiological knowledge of the organism. As prediction outside the experimental region is a significant benefit of mechanistic models [56], it is important that the reliability of such predictions is established, as demonstrated in the current contribution. Furthermore, considering the subsequent output variance (Figure 5A) associated with the parameter estimation in Figure 2 in the case study, it is apparent that the mere fitting of a model to experimental data (and even validation with similar experimental data) is not enough to guarantee the accuracy and reliability of future predictions. Unfortunately, as pointed out by Sin and co-workers [12] only a few mechanistic models are reported in the literature with reliability analysis. In more intricate applications, such as in using models to elucidate metabolic activity [57], to derive further understanding from molecular level interactions [58] and in soft sensor design [59], model reliability is of key importance. Therefore, it is imperative that the parameters of such models are estimated to very narrow confidence intervals by thoroughly analysing their identifiability and applying the proper regularization technique, so as not to produce misleading biological behaviour in their predictions. 
Performing identifiability tests for large non-linear models can be a daunting task, with requirements of special mathematical understanding [19] and high computational power. However, we hope that the simplified treatments given here will appeal to biotechnologists and bioprocess engineers and hence promote a more thorough handling of dynamical models, even for non-experienced modellers. This will extend the use of mechanistic models and accelerate the digital revolution in biotechnology.

\section{ACKNOWLEDGEMENTS}

We are grateful for financial support from the European Union's Horizon 2020 research and innovation program under the Marie Skłodowska-Curie actions grant agreement No. 643056 (Biorapid).

\section{Conflict of Interest}

The authors declare no financial or commercial conflict of interest.

\section{Supplementary Data}

The Matlab ${ }^{\circledR}$ code of the model analysis framework and the fermentation data used in the case study can be found at: https://gitlab.tubit.tuberlin.de/nicolas.cruz/E_coli_fed-batch/tree/master/Anane_2019b_BEJ 


\section{References}

[1] J. Monod, The Growth of Bacterial Cultures, Annu. Rev. Microbiol. 3 (1949) 371-394.

[2] J.F. Andrews, A mathematical model for the continuous culture of microorganisms utilizing inhibitory substrates, Biotechnol. Bioeng. 10 (1968) 707-723. doi:10.1002/bit.260100602.

[3] D.J. Batstone, J. Keller, I. Angelidaki, S. V. Kalyuzhnyi, S.G. Pavlostathis, A. Rozzi, W.T. Sanders, H. Siegrist, V.A. Vavilin, The IWA Anaerobic Digestion Model No 1 (ADM1),, Water Sci. Technol. 45 (2002) 65-73. doi:10.2166/wst.2008.678.

[4] M.A. Savageau, Biochemical systems analysis. III. Dynamic solutions using a power-law approximation, J. Theor. Biol. 26 (1970) 215-226. doi:10.1016/S0022-5193(70)80013-3.

[5] H. Kitano, Computational systems biology, Nature. 420 (2002) 206-210. doi:10.1038/nature01254.

[6] G. Stephanopoulos, A. Aristidou, J. Nielsen, Metabolic Engineering: Principles and Methodologies, Academic Press, San Diego, 1998. doi:10.1016/B978012666260-3/50003-0.

[7] A. Varma, B.O. Palsson, Metabolic flux balancing: Basic concepts, scientific and practical use, Bio/Technology. 12 (1994) 994-998. doi:10.1038/nbt1094994.

[8] M.A. Marchisio, J. Stelling, Computational design tools for synthetic biology, Curr. Opin. Biotechnol. 20 (2009) 479-485. doi:10.1016/j.copbio.2009.08.007.

[9] Y. Saeys, I. Inza, P. Larrañaga, A review of feature selection techniques in bioinformatics, $\quad$ Bioinformatics. $\quad 23 \quad$ (2007) 2507-2517. doi:10.1093/bioinformatics/btm344.

[10] J.E. Bailey, Mathematical modeling and analysis in biochemical engineering: Past accomplishments and future opportunities, Biotechnol. Prog. 14 (1998) 8- 
20. doi:10.1021/bp9701269.

[11] M. Koutinas, A. Kiparissides, E.N. Pistikopoulos, A. Mantalaris, Bioprocess Systems Engineering: Transferring Traditional Process Engineering Principles To Industrial Biotechnology, Comput. Struct. Biotechnol. J. 3 (2012) 1-9. doi:10.5936/csbj.201210022.

[12] G. Sin, K. V Gernaey, A.E. Lantz, Good modelling practice (GMoP) for PAT applications: Propagation of input uncertainty and sensitivity analysis, Biotechnol. Prog. 25 (2009) 1043-1053. doi:10.1021/bp.166.

[13] A. Raue, C. Kreutz, T. Maiwald, J. Bachmann, M. Schilling, U. Klingmüller, J. Timmer, Structural and practical identifiability analysis of partially observed dynamical models by exploiting the profile likelihood, Bioinformatics. 25 (2009) 1923-1929. doi:10.1093/bioinformatics/btp358.

[14] C. Kravaris, J. Hahn, Y. Chu, Advances and selected recent developments in state and parameter estimation, Comput. Chem. Eng. 51 (2013) 111-123. doi:10.1016/j.compchemeng.2012.06.001.

[15] R. Brun, K. Martin, H. Siegrist, W. Gujer, P. Reichert, Practical identifiability of ASM2d parameters - systematic selection and tuning of parameter subsets, Water Res. 36 (2002) 4113-4127.

[16] Y. Bard, Non-linear parameter estimation, Academic Press, New York, 1974.

[17] E. Balsa-Canto, D. Henriques, A. Gábor, J.R. Banga, AMIGO2, a toolbox for dynamic modeling, optimization and control in systems biology, Bioinformatics. 32 (2016) 3357-3359. doi:10.1093/bioinformatics/btw411.

[18] A.F. Villaverde, D. Henriques, K. Smallbone, S. Bongard, J. Schmid, D. CicinSain, A. Crombach, J. Saez-Rodriguez, K. Mauch, E. Balsa-Canto, P. Mendes, J. Jaeger, J.R. Banga, BioPreDyn-bench: a suite of benchmark problems for dynamic modelling in systems biology., BMC Syst. Biol. 9 (2015) 8. doi:10.1186/s 12918-015-0144-4.

[19] R. Muñoz-Tamayo, L. Puillet, J.B. Daniel, D. Sauvant, O. Martin, M. Taghipoor, 
P. Blavy, Review: To be or not to be an identifiable model. Is this a relevant question in animal science modelling?, Animal. 12 (2018) 701-712. doi:10.1017/S1751731117002774.

[20] A.F. Villaverde, A. Barreiro, A. Papachristodoulou, Structural Identifiability of Dynamic Systems Biology Models, PLoS Comput. Biol. 12 (2016) 1-22. doi:10.1371/journal.pcbi.1005153.

[21] J. Almquist, M. Cvijovic, V. Hatzimanikatis, J. Nielsen, M. Jirstrand, Kinetic models in industrial biotechnology - Improving cell factory performance, Metab. Eng. 24 (2014) 38-60. doi:10.1016/j.ymben.2014.03.007.

[22] M.P. Saccomani, Structural vs Practical Identifiability in System Biology, IWBBIO 2013 Proc. (2013) 305-313.

[23] G. Franceschini, S. Macchietto, Model-based design of experiments for parameter precision : State of the art, Chem. Eng. Sci. 63 (2008) 4846-4872. doi:10.1016/j.ces.2007.11.034.

[24] N. Vora, P. Daoutidis, Nonlinear model reduction of chemical reaction systems, AIChE J. 47 (2001) 2320-2332. doi:10.1002/aic.690471016.

[25] A. Raue, C. Kreutz, T. Maiwald, U. Klingmuller, J. Timmer, Addressing parameter identifiability by model-based experimentation, IET Syst. Biol. 5 (2011) 120-130. doi:10.1049/iet-syb.2010.0061.

[26] T.A. Johansen, On Tikhonov regularization, bias and variance in nonlinear system identification, Automatica. 33 (1997) 441-446. doi:10.1016/S00051098(96)00168-9.

[27] D.C. López C., T. Barz, S. Körkel, G. Wozny, Nonlinear ill-posed problem analysis in model-based parameter estimation and experimental design, Comput. Chem. Eng. 77 (2015) 24-42. doi:10.1016/j.compchemeng.2015.03.002.

[28] O.T. Chis, J.R. Banga, E. Balsa-Canto, Structural identifiability of systems biology models: A critical comparison of methods, PLoS One. 6 (2011). doi:10.1371/journal.pone.0027755. 
[29] T. Quaiser, M. Mönnigmann, Systematic identifiability testing for unambiguous mechanistic modeling--application to JAK-STAT, MAP kinase, and NF-kappaB signaling pathway models., BMC Syst. Biol. 3 (2009) 50. doi:10.1186/17520509-3-50.

[30] D. Brockmann, K.H. Rosenwinkel, E. Morgenroth, Practical identifiability of biokinetic parameters of a model describing two-step nitrification in biofilms, Biotechnol. Bioeng. 101 (2008) 497-514. doi:10.1002/bit.21932.

[31] R. Lencastre Fernandes, V.K. Bodla, M. Carlquist, A.-L. Heins, A. Eliasson Lantz, G. Sin, K. V. Gernaey, Applying Mechanistic Models in Bioprocess Development, in: Adv. Biochem. Eng. Biotechnol., 2012: pp. 137-166. doi:10.1007/10_2012_166.

[32] D.A. Belsley, E. Kuh, R.E. Welsch, Regression Diagnostics: Identifying Influential Data and Sources of Collinearity, John Wiley \& Sons, Inc., Hoboken, NJ, USA, 1980. doi:10.1002/0471725153.

[33] S.R. Weijers, P.A. Vanrolleghem, A procedure for selecting best identifiable parameters in calibrating Activated Sludge Model No. 1 to full-scale plant data, Water Sci. Technol. 36 (1997) 69-79. doi:10.1016/S0273-1223(97)00463-0.

[34] a Guisasola, J. a Baeza, J. Carrera, G. Sin, P. a Vanrolleghem, J. Lafuente, F.I. Matrix, The influence of experimental data quality and quantity on parameter estimation accuracy. Andrews Inhibition Model as a Case Study, Eduacation Chem. Eng. 1 (2006) 139-145. doi:10.1205/ece06016.

[35] D.J.W. De Pauw, P. a Vanrolleghem, Practical aspects of sensitivity function approximation for dynamic models, Math. Comput. Model. Dyn. Syst. 12 (2006) 395-414. doi:10.1080/13873950600723301.

[36] A. Gábor, J.R. Banga, Robust and efficient parameter estimation in dynamic models of biological systems, BMC Syst. Biol. 9 (2015). doi:10.1186/s12918015-0219-2.

[37] G. Sin, K. V. Gernaey, Data Handling and Parameter Estimation, in: M.C.. van Loosdrecht, P.H. Nielsen, C.M. Lopez-Vazquez, D. Brdjanovic (Eds.), Exp. 
Methods Wastewater Treat., 1st ed., IWA Publishing, London, 2016: pp. 201234.

[38] G.H. Golub, C.F. Van Loan, Matrix Computations, 3rd ed., The Johns Hopkins University Press, Baltimore, 1996. doi:10.1063/1.3060478.

[39] T.F. Chan, P.C. Hansen, Some Applications of the Rank Revealing QR Factorization, SIAM J. Sci. Stat. Comput. 13 (1992) 727-741. doi:10.1137/0913043.

[40] D.C. López, G. Wozny, A. Flores-Tlacuahuac, R. Vasquez-Medrano, V.M. Zavala, A Computational Framework for Identifiability and Ill-Conditioning Analysis of Lithium-Ion Battery Models, Ind. Eng. Chem. Res. 55 (2016) 30263042. doi:10.1021/acs.iecr.5b03910.

[41] M.N. Cruz Bournazou, H. Arellano-Garcia, G. Wozny, G. Lyberatos, C. Kravaris, ASM3 extended for two-step nitrification-denitrification: a model reduction for sequencing batch reactors, J. Chem. Technol. Biotechnol. 87 (2012) 887-896. doi:10.1002/jctb.3694.

[42] R. Milo, P. Jorgensen, U. Moran, G. Weber, M. Springer, BioNumbers--the database of key numbers in molecular and cell biology., Nucleic Acids Res. 38 (2010) D750-3. doi:10.1093/nar/gkp889.

[43] I. Schomburg, A. Chang, O. Hofmann, C. Ebeling, F. Ehrentreich, D. Schomburg, BRENDA: A resource for enzyme data and metabolic information, Trends Biochem. Sci. 27 (2002) 54-56. doi:10.1016/S0968-0004(01)02027-8.

[44] F. Campolongo, Sensitivity analysis of an environmental model: an application of different analysis methods, Reliab. Eng. Syst. Saf. 57 (1997) 49-69. doi:10.1016/S0951-8320(97)00021-5.

[45] R.L. Iman, W.J. Conover, A Measure of Top - Down Correlation, Technometrics. 29 (1987) 351-357. doi:10.1080/00401706.1987.10488244.

[46] T.A. Mara, S. Tarantola, P. Annoni, Non-parametric methods for global sensitivity analysis of model output with dependent inputs, Environ. Model. 
Softw. 72 (2015) 173-183. doi:10.1016/j.envsoft.2015.07.010.

[47] P. Neubauer, H.Y. Lin, B. Mathiszik, Metabolic load of recombinant protein production: Inhibition of cellular capacities for glucose uptake and respiration after induction of a heterologous gene in Escherichia coli, Biotechnol. Bioeng. 83 (2003) 53-64. doi:10.1002/bit.10645.

[48] H.T.B. Pham, G. Larsson, S.O. Enfors, Growth and energy metabolism in aerobic fed-batch cultures of Saccharomyces cerevisiae: Simulation and model verification, Biotechnol. Bioeng. 60 (1998) 474-482. doi:10.1002/(SICI)10970290(19981120)60:4<474::AID-BIT9>3.0.CO;2-J.

[49] B. Xu, M. Jahic, S.-O. Enfors, Modeling of Overflow Metabolism in Batch and Fed-Batch Cultures of Escherichia coli, Biotechnol. Prog. 15 (1999) 81-90. doi:10.1021/bp9801087.

[50] M.N. Cruz Bournazou, T. Barz, D.B. Nickel, D.C. Lopez Cárdenas, F. Glauche, A. Knepper, P. Neubauer, Online optimal experimental re-design in robotic parallel fed-batch cultivation facilities, Biotechnol. Bioeng. 114 (2017) 610619. doi:10.1002/bit.26192.

[51] E. Anane, D.C. López C, P. Neubauer, M.N. Cruz Bournazou, Modelling overflow metabolism in Escherichia coli by acetate cycling, Biochem. Eng. J. 125 (2017) 23-30. doi:10.1016/j.bej.2017.05.013.

[52] D.B. Nickel, M.N. Cruz-Bournazou, T. Wilms, P. Neubauer, A. Knepper, Online bioprocess data generation, analysis, and optimization for parallel fed-batch fermentations in milliliter scale, Eng. Life Sci. 17 (2017) 1195-1201. doi:10.1002/elsc.201600035.

[53] E. Anane, E. van Rensburg, J.F. Görgens, Optimisation and scale-up of $\alpha$ glucuronidase production by recombinant Saccharomyces cerevisiae in aerobic fed-batch culture with constant growth rate, Biochem. Eng. J. 81 (2013) 1-7. doi:10.1016/j.bej.2013.09.012.

[54] B. Xu, M. Jahic, G. Blomsten, S.O. Enfors, Glucose overflow metabolism and mixed-acid fermentation in aerobic large-scale fed-batch processes with 
Escherichia coli, Appl. Microbiol. Biotechnol. 51 (1999) 564-571. doi:10.1007/s002530051433.

[55] H.Y. Lin, B. Mathiszik, B. Xu, S.-O.O. Enfors, P. Neubauer, Determination of the maximum specific uptake capacities for glucose and oxygen in glucoselimited fed-batch cultivations of Escherichia coli, Biotechnol. Bioeng. 73 (2001) 347-357. doi:10.1002/bit.1068.

[56] L. Mears, S.M. Stocks, M.O. Albaek, G. Sin, K. V. Gernaey, Mechanistic Fermentation Models for Process Design, Monitoring, and Control, Trends Biotechnol. 35 (2017) 914-924. doi:10.1016/j.tibtech.2017.07.002.

[57] A. Chowdhury, A. Khodayari, C.D. Maranas, Improving prediction fidelity of cellular metabolism with kinetic descriptions, Curr. Opin. Biotechnol. 36 (2015) 57-64. doi:10.1016/j.copbio.2015.08.011.

[58] J. Stelling, Mathematical models in microbial systems biology, Curr. Opin. Microbiol. 7 (2004) 513-518. doi:10.1016/j.mib.2004.08.004.

[59] R. Spann, C. Roca, D. Kold, A. Eliasson Lantz, K. V. Gernaey, G. Sin, A probabilistic model-based soft sensor to monitor lactic acid bacteria fermentations, Biochem. Eng. J. $135 \quad$ (2018) 49-60. doi:10.1016/j.bej.2018.03.016.

[60] P.C. Hansen, Analysis of Discrete Ill-Posed Problems by Means of the L-Curve, SIAM Rev. 34 (1992) 561-580. doi:10.1137/1034115.

[61] H.Y. Lin, B. Mathiszik, B. Xu, S.O. Enfors, P. Neubauer, Determination of the maximum specific uptake capacities for glucose and oxygen in glucose-limited fed-batch cultivations of Escherichia coli, Biotechnol. Bioeng. 73 (2001) 347357. doi:10.1002/bit.1068.

[62] K. Martínez-Gómez, N. Flores, H.M. Castañeda, G. Martínez-Batallar, G. Hernández-Chávez, O.T. Ramírez, G. Gosset, S. Encarnación, F. Bolivar, New insights into Escherichia coli metabolism: carbon scavenging, acetate metabolism and carbon recycling responses during growth on glycerol., Microb. Cell Fact. 11 (2012) 46. doi:10.1186/1475-2859-11-46. 


\section{Figure legends}

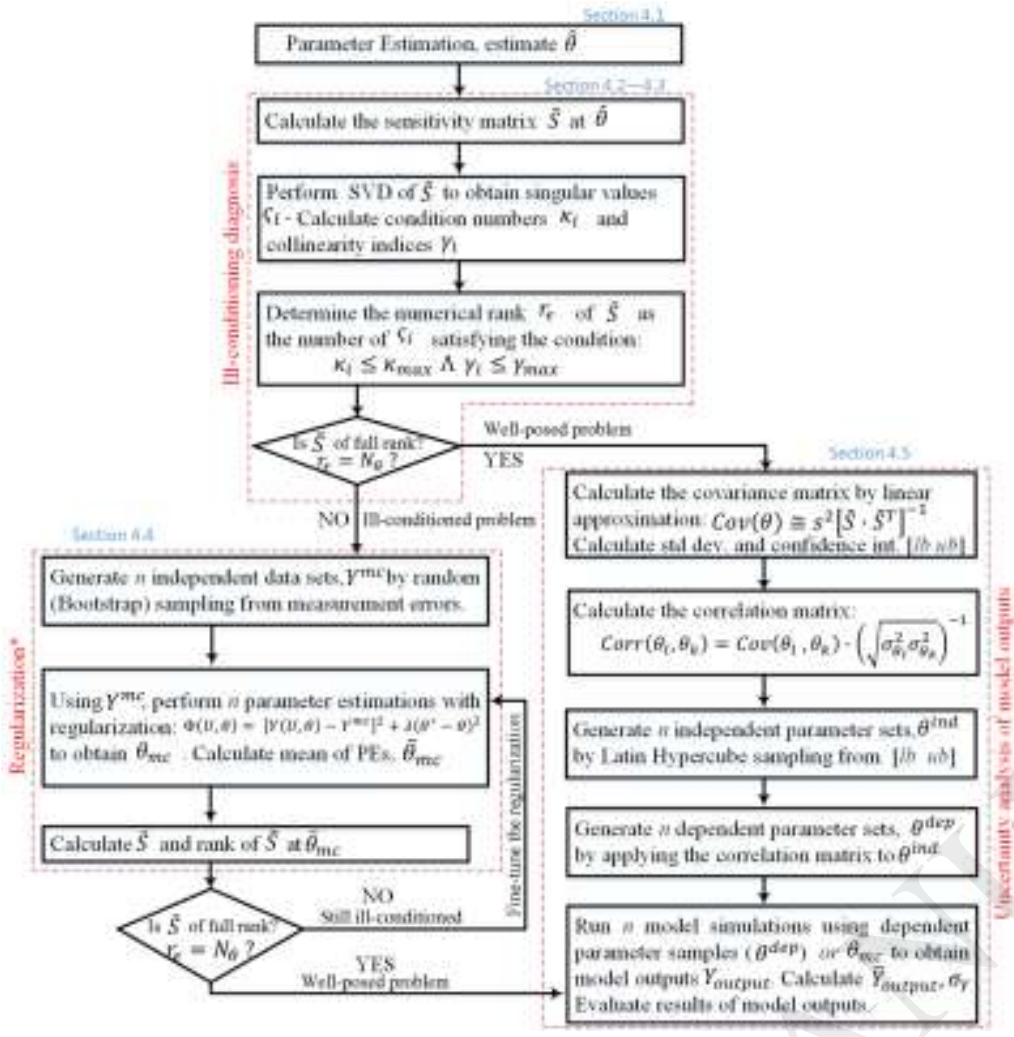

Figure 1 Framework for ill-conditioning diagnosis, regularization and uncertainty analyses of mechanistic growth models. SVD $=$ Singular value decomposition. The thresholds $\kappa_{\max }$ and $\gamma_{\max }$ are empirical coefficients. *An alternative method of regularization, namely Subset Selection $(\mathrm{SsS})$ is presented in the Supplementary material.
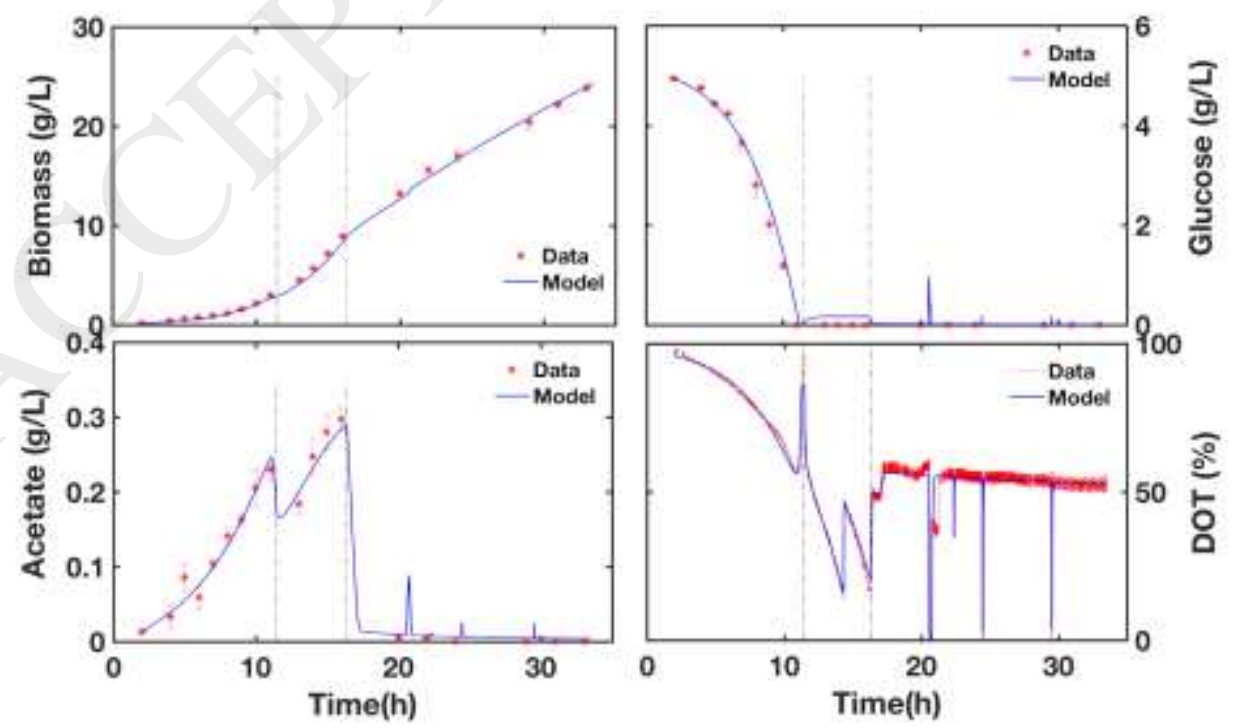
Figure 2 Model fitting of fed-batch fermentation of wild type E. coli W3110. Dashed lines signify the beginning and end of exponential feed fed-batch phase. Adapted from [51]

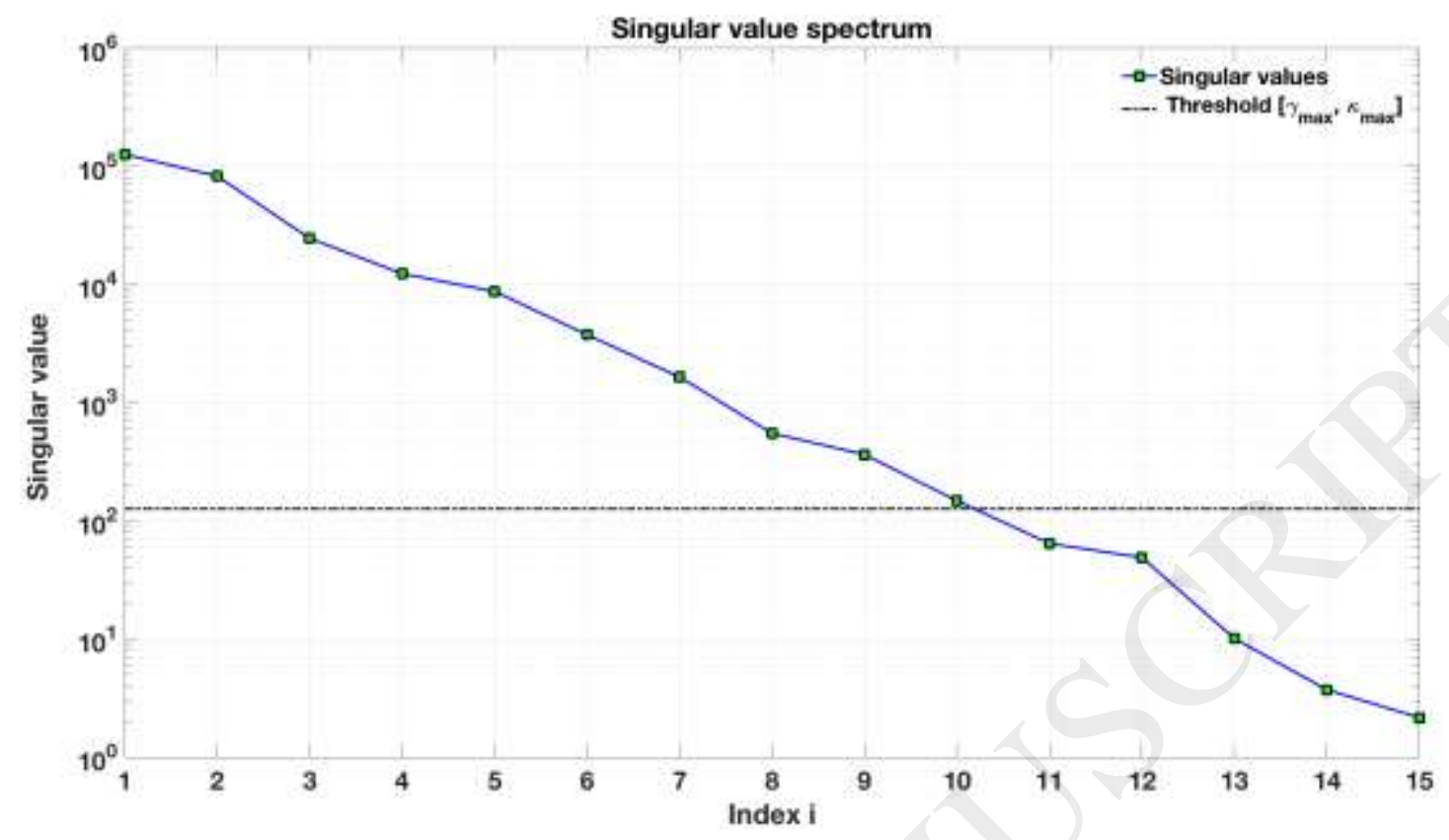

Figure 3 Singular value spectrum of the sensitivity matrix showing the ill-conditioned parameter estimation problem, based on the thresholds for the numerical rank $r_{\epsilon}$ of $\tilde{S}$. The number of singular values above the threshold line is the numerical rank, in this case $r_{\epsilon}=10$. 
A
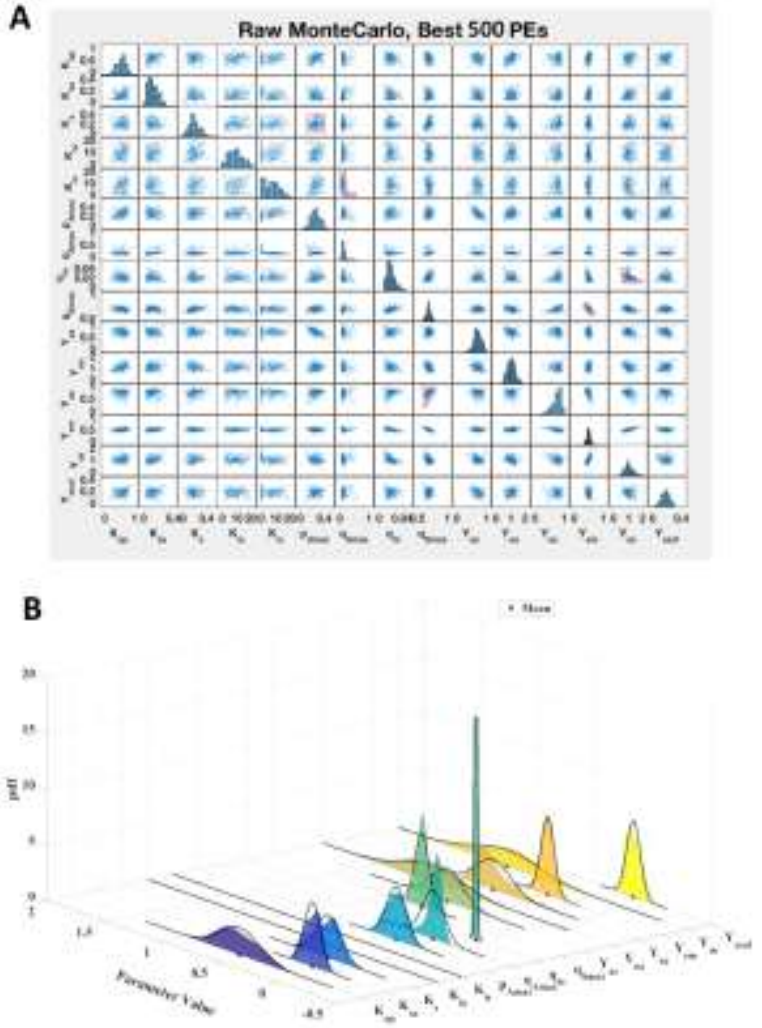

Figure 4 (A) Matrix plot of the non-regularized parameter estimation results showing a few of the inherent non-linearly correlated $\left(q_{\text {Amax }}\right.$ and $K_{i s} ; Y_{o s}$ and $\left.q_{m}\right)$, linearly correlated $\left(q_{\text {Smax }}\right.$ and $\left.Y_{e m}\right)$ and non-correlated $\left(p_{A \max }\right.$ and $\left.K_{s}\right)$ parameters of the model. (B) Probability distribution of the Monte Carlo parameter estimates showing the real distributions (coloured inlay) and the assumed normal distribution which was used for subsequent output uncertainty analysis. 

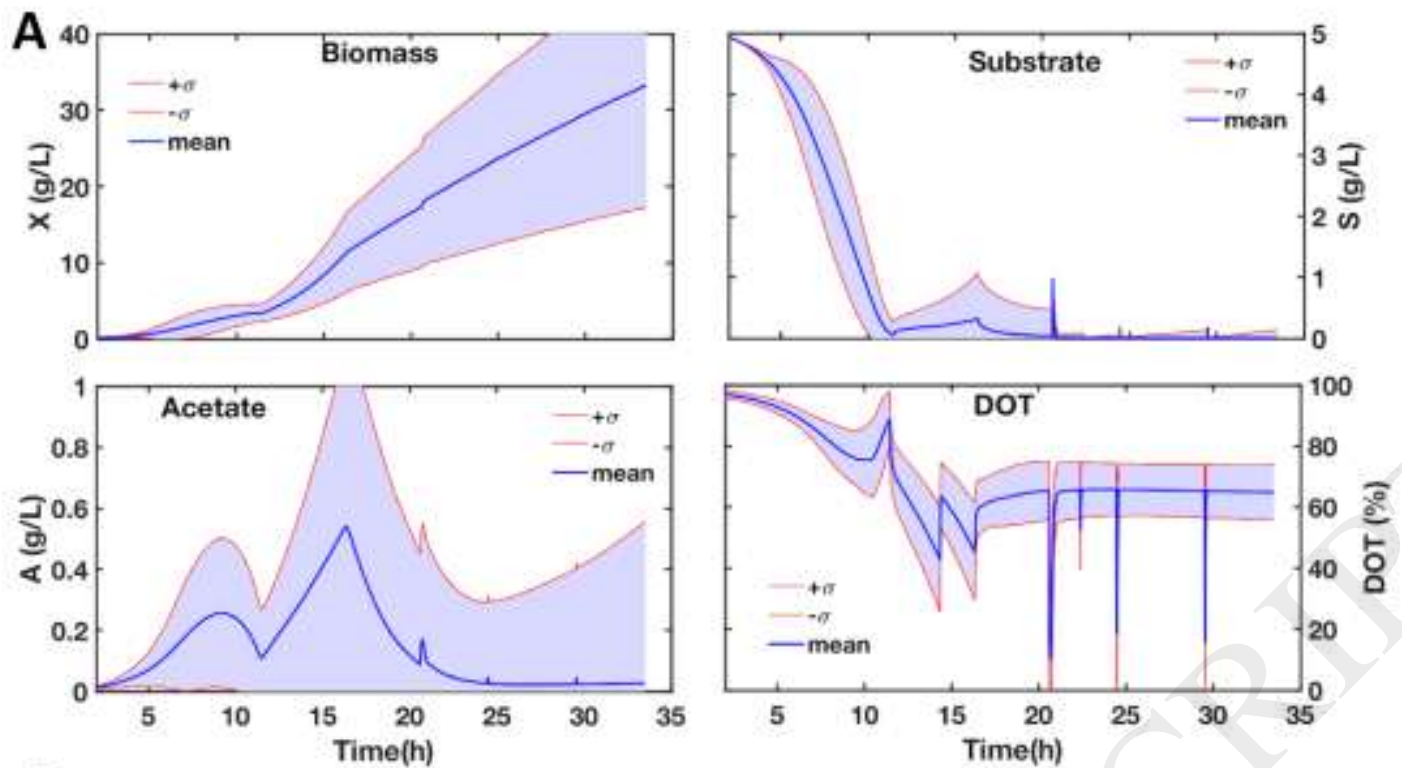

B
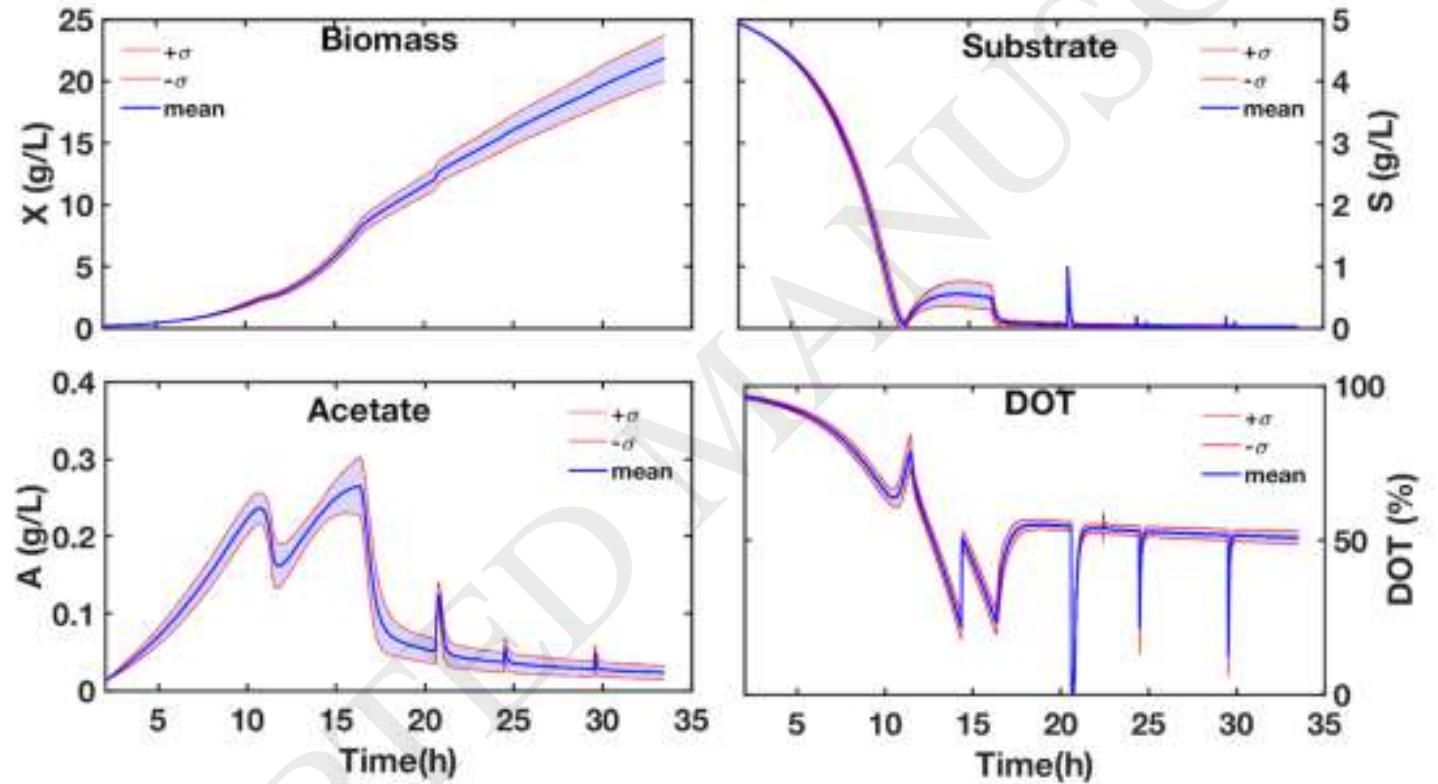

Figure 5 Output uncertainty analysis with (A) non-regularized and (B) regularized Monte Carlo parameter estimates (500 samples). Outer envelops signify one standard deviation from the mean (middle line). 

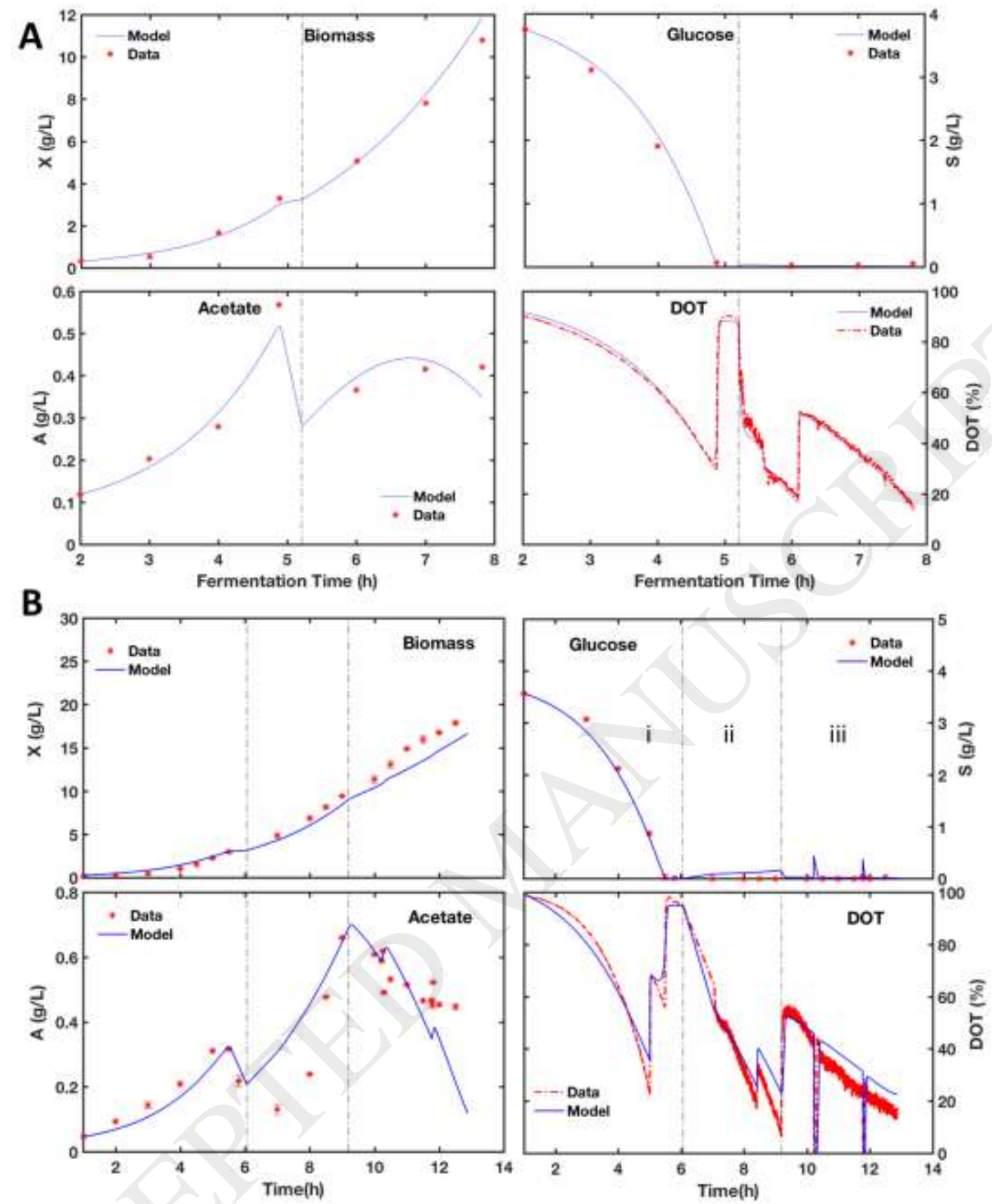

Figure 6 Validation of mechanistic model of E. coli after regularized parameter estimation using two independent cultivations, $\mathrm{A}$ and $\mathrm{B}$. In $\mathrm{B}, \mathrm{i}-$ batch phase, $\mathrm{ii}-$ exponential feed fed-batch at $\mu_{\text {set }}=0.32 \mathrm{~h}^{-1}$, iii-exponential feed fed-batch at $\mu_{\text {set }}=$ $0.15 \mathrm{~h}^{-1}$ with intermittent glucose pulses. 


\section{Tables}

Table 1 Results of parameter estimation for wild type strain of E. coli W3110 (with Tikhonov regularization). Also shown is the quantification of the uncertainty associated with the parameter estimators. LB-lower bound, UB-upper bounds, CI-95\% confidence interval.

\begin{tabular}{|c|c|c|c|c|c|c|}
\hline \multirow[t]{2}{*}{ Parameter $^{+}$} & \multirow[t]{2}{*}{ Units } & \multirow{2}{*}{$\begin{array}{l}\text { Initial guess } \\
\text { (literature)* }\end{array}$} & \multirow[t]{2}{*}{ Estimate } & \multicolumn{3}{|c|}{ PE uncertainty quantification } \\
\hline & & & & $\% \sigma_{\theta}$ & LB-CI & UB-CI \\
\hline $\mathrm{K}_{\mathrm{ap}}$ & $\mathrm{g} \mathrm{g}^{-1} \mathrm{~h}^{-1}$ & 0.10 & 0.5031 & 15.2 & 0.3539 & 0.6565 \\
\hline $\mathrm{K}_{\mathrm{sa}}$ & $\mathrm{g} \mathrm{L}^{-1}$ & 0.05 & 0.0131 & 22.0 & 0.0076 & 0.0192 \\
\hline $\mathrm{K}_{\mathrm{o}}$ & $\mathrm{g} \mathrm{L}^{-1}$ & 10.0 & 0.0001 & 0.0 & 0.0001 & 0.0001 \\
\hline $\mathrm{K}_{\mathrm{s}}$ & $\mathrm{g} \mathrm{L}^{-1}$ & 0.05 & 0.0370 & 8.9 & 0.0305 & 0.0435 \\
\hline $\mathrm{K}_{\mathrm{ia}}$ & $\mathrm{g} \mathrm{L}^{-1}$ & 5.00 & 1.2293 & 9.6 & 1.0062 & 1.4737 \\
\hline $\mathrm{K}_{\mathrm{is}}$ & $\mathrm{g} \mathrm{L}^{-1}$ & 10.0 & 2.1231 & 27.3 & 0.9788 & 3.2673 \\
\hline $\mathrm{p}_{\text {Amax }}$ & $\mathrm{g} \mathrm{g}^{-1} \mathrm{~h}^{-1}$ & 0.17 & 0.2268 & 6.5 & 0.1977 & 0.2558 \\
\hline $\mathrm{q}_{\text {Amax }}$ & $\mathrm{g} \mathrm{g}^{-1} \mathrm{~h}^{-1}$ & 0.15 & 0.1148 & 6.1 & 0.1009 & 0.1287 \\
\hline $\mathrm{q}_{\mathrm{m}}$ & $\mathrm{g} \mathrm{g}^{-1} \mathrm{~h}^{-1}$ & 0.04 & 0.0129 & 7.0 & 0.0111 & 0.0147 \\
\hline $\mathrm{q}_{\operatorname{Smax}}$ & $\mathrm{g} \mathrm{g}^{-1} \mathrm{~h}^{-1}$ & 1.37 & 0.6321 & 0.3 & 0.6320 & 0.6392 \\
\hline $\mathrm{Y}_{\mathrm{as}}$ & $\mathrm{g} \mathrm{g}^{-1}$ & 0.80 & 0.9097 & 4.5 & 0.8283 & 0.9911 \\
\hline$Y_{\text {oa }}$ & $\mathrm{g} \mathrm{g}^{-1}$ & 1.06 & 0.5440 & 9.5 & 0.4425 & 0.6455 \\
\hline$Y_{x a}$ & $\mathrm{~g} \mathrm{~g}^{-1}$ & 0.70 & 0.5718 & 9.9 & 0.4604 & 0.6833 \\
\hline $\mathrm{Y}_{\mathrm{em}}$ & $\mathrm{g} \mathrm{g}^{-1}$ & 0.50 & 0.5333 & 2.4 & 0.5085 & 0.5580 \\
\hline$Y_{\mathrm{os}}$ & $\mathrm{g} \mathrm{g}^{-1}$ & 1.06 & 1.5620 & 5.4 & 1.3941 & 1.7298 \\
\hline$Y_{x s o f}$ & $\mathrm{~g} \mathrm{~g}^{-1}$ & 0.15 & 0.2268 & 12.0 & 0.1730 & 0.2807 \\
\hline
\end{tabular}

\footnotetext{
${ }^{+}$Parameter descriptions in nomenclature. * References: $[47,49,61,62]$
} 